\title{
Different kind of textures of Yukawa coupling matrices in the two Higgs doublet model type III
}

\author{
A. E. Cárcame* \\ Departamento de Física, \\ Universidad Nacional de Colombia, Bogotá, Colombia \\ Scuola Normale Superiore Di Pisa, Pisa, Italy \\ R. Martíned and J.-Alexis Rodríguedt \\ Departamento de Física, Universidad Nacional de Colombia \\ Bogotá, Colombia
}

(Dated:)

\begin{abstract}
The quark mass matrices ansatze proposed by Fritzsch, Du-Xing and Fukuyama-Nishiura in the framework of the general two Higgs doublet model are studied. The corresponding Yukawa matrices in the flavor basis in the different cases considered are discussed. The corresponding Cabbibo-Kobayashi-Maskawa matrix elements are computed in all cases and compared with their experimental values. The complex phases of the ansatze are taken into account and the CP violating phase $\delta$ is computed. Finally, in order to observe the influence of the different kind of textures of Yukawa coupling matrices considered, some phenomenology of two body decays of the top quark, the lightest Higgs boson and the charged Higgs boson is discussed.
\end{abstract}

\section{INTRODUCTION}

Despite of all its success, the standard model (SM) of electroweak interactions based on the $S U(3)_{C} \otimes S U(2)_{L} \otimes U(1)_{Y}$ gauge symmetry has many unexplained features. Most of them are linked to the fermionic sector, such as the origin of the fermion masses and the mixing angles [1, 2, 3, 4]. These reasons lead to consider that the SM is not the most fundamental theory of the basic interactions. It should be considered as an effective theory that remains valid up to some energy scale of the order of $\mathrm{TeV}$ and eventually it will be replaced by a more fundamental theory. In the framework of the SM, the values of the Yukawa couplings are parameterized in a phenomenological way. The quark masses and mixing matrix are described by 10 free parameters: six quark masses, three flavor mixing angles and one $\mathrm{CP}$ violating phase. It is, the form of the Yukawa couplings is not well understood and neither their origin nor the underlying principles, which it is known as the flavor problem. Attempts to compute these 10 phenomenological parameters have been done within the framework of the extensions of the SM including grand unification theories, supersymmetric theories and superstring theories [5, 6, 7]. There are two basic approaches to study the patterns of Yukawa couplings: one way is adopting the unification hypothesis of matter multiplets and the second one is to assume a specific form of the Yukawa couplings called texture [8]. The two approaches are close related because the mass matrices gotten using textures could be incorporated into the grand unified theories [5, 9]. The use of quark mass matrix textures is motivated by the observed large hierarchies of the quark masses and the Cabbibo-KobayashiMaskawa (CKM) matrix elements [10, 11, 13, 14]. Phenomenological quark mass matrices have been discussed from different points of view. For example, quark mass matrix ansatze have been used in an analogous way in the leptonic sector, trying to get the lepton mixing matrix in order to explain the neutrino anomalies [9, 14, 15, 16]. Also, new texture ansatze have been proposed motivated by more precise results on the elements of the quark mixing matrix [1, 10, 11, 17]. Quark and lepton mass matrices have also been discussed in the context of the $S O(10)$ Grand Unification theories [9]. The understanding of the discrete flavor symmetries hidden in such textures may be useful in the knowledge of the underlying dynamics responsible for quark mass generation and CP violation.

One possible simple extension of the SM is by adding a new Higgs doublet and it is called the two Higgs Doublet Model (2HDM). This extension has the following direct consequences: it increases the scalar spectrum and it gives a more generic pattern of the Flavor Changing Neutral Currents (FCNC). FCNC at tree level can be consider a problem that was solved in the earlier versions of the two Higgs Doublet Model (2HDM type I and II) by imposing a discrete symmetry that restricts each fermion to be coupled at most to one Higgs doublet [18]. But if the discrete symmetry is

\footnotetext{
*Electronic address: aecarcamoh@unal.edu.co,antonio.carcamo@sns.it

${ }^{\dagger}$ Electronic address: remartinezm@unal.edu.co

${ }^{\ddagger}$ Electronic address: jarodriguezl@unal.edu.co
} 
not imposed then FCNC at tree level remains, it is the so-called two Higgs Doublet Model type III. The 2HDM type III and its phenomenology have been extensively studied in the literature [19]. In the 2HDM-III, for each type quark, up or down type, there are two Yukawa couplings. One of the Yukawa couplings is in charge of generating the quark masses and the other one produces the flavor changing couplings at tree level. But in any case both Higgs doublets have the same quantum numbers then if one ansatz is assumed for the Yukawa coupling which generates the quark masses, is valid to associate the same structure for the other Yukawa coupling which is generating the flavor changing couplings, it is the main hypothesis of this work.

In this manuscript, different kind of textures of Yukawa coupling matrices are discussed in the framework of the $2 \mathrm{HDM}$ type III. In section【, the principal features of the $2 \mathrm{HDM}$ type III are reviewed, then in section III the different kind of texture ansatze are introduced. First of all, the Fritzsch ansatz and the Du-Xing ansatz are reviewed. Then, the Fritzsch and Du-Xing ansatze are combined, searching a better agreement with the experimental results. A brief review of the Fukuyama-Nishiura mass matrix ansatz is presented and finally a new ansatz is proposed. The new ansatz presents the feature that the top quark does not have any mix and therefore it does not have any flavor changing neutral process at tree level. The corresponding Yukawa coupling matrices in the mass basis in the framework of the 2HDM type III are gotten for all the ansatze discussed, it means the intensity of couplings that lead to FCNC processes are obtained. Also, the numerical values of the corresponding CKM matrix elements are obtained. In section IV] some phenomenological aspects of the two body decays of the top quark, the lightest Higgs boson and the charged Higgs boson are discussed. Finally in section $\mathrm{V}$ our conclusions are presented.

\section{YUKAWA INTERACTION LAGRANGIAN FOR THE TWO HIGGS DOUBLET MODEL}

In the most general form of the two Higgs doublet model (2HDM), the Lagrangian for the Yukawa interaction in the quark sector is given by [20],

$$
-L_{Y}=\bar{q}_{L}^{0} \eta^{U, 0} \tilde{\phi}_{1} U_{R}^{0}+\bar{q}_{L}^{0} \eta^{D, 0} \phi_{1} D_{R}^{0}+\bar{q}_{L}^{0} \xi^{U, 0} \tilde{\phi}_{2} U_{R}^{0}+\bar{q}_{L}^{0} \xi^{D, 0} \phi_{2} D_{R}^{0}+h . c
$$

where $\eta^{f, 0}$ and $\xi^{f, 0}$ are the Yukawa interaction matrices being $\tilde{\phi}=i \tau_{2} \phi^{*}, f^{0}=U^{0}, D^{0}, q_{L}^{0}=\left(U^{0}, D^{0}\right)_{L}$ the quark doublet states in the interaction basis, and $U^{0}=\left(u^{0}, c^{0}, t^{0}\right), D^{0}=\left(d^{0}, s^{0}, b^{0}\right)$ the quark interaction eigenstates. The two Higgs boson fields, after spontaneous symmetry breaking, have the following form:

$$
\phi_{k}=\left(\begin{array}{c}
\phi_{k}^{+} \\
\frac{1}{\sqrt{2}}\left(v_{k}+\phi_{k}^{0}+i \lambda_{k}^{0}\right)
\end{array}\right) \quad \text { with } \quad k=1,2
$$

where $v_{1}$ and $v_{2}$ are the vacuum expectation values of the two Higgs fields $\phi_{1}$ and $\phi_{2}$, respectively. There are not any extra discrete symmetry acting on the Higgs fields, it means they can mix because they have the same quantum numbers. Furthermore, both Higgs doublets can give mass to down- and up-type quarks simultaneously. However, the Higgs fields can be rotated to a new base where

$$
\left(\begin{array}{l}
\phi_{1}^{\prime} \\
\phi_{2}^{\prime}
\end{array}\right)=\left(\begin{array}{cc}
\cos \beta & \sin \beta \\
-\sin \beta & \cos \beta
\end{array}\right)\left(\begin{array}{l}
\phi_{1} \\
\phi_{2}
\end{array}\right)
$$

in such case $\tan \beta=v_{2} / v_{1}$ and the vacuum expectation values are $\left\langle\phi_{1}^{\prime}\right\rangle=v=\sqrt{v_{1}^{2}+v_{2}^{2}}$ and $\left\langle\phi_{2}^{\prime}\right\rangle=0$. In this article, we are not considering spontaneous CP symmetry breaking in the Higgs sector. In the new mentioned base,

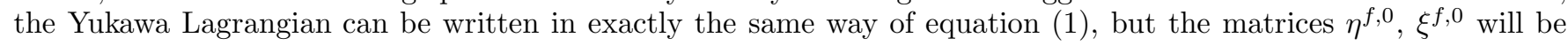
rotated to a new base

$$
\left(\begin{array}{l}
\eta^{\prime} f, 0 \\
\xi^{\prime} f, 0
\end{array}\right)=\left(\begin{array}{cc}
\cos \beta & -\sin \beta \\
\sin \beta & \cos \beta
\end{array}\right)\left(\begin{array}{l}
\eta^{f, 0} \\
\xi^{f, 0}
\end{array}\right)
$$

In general the quantum system will be in the prime base where only one vacuum expectation value is different from zero [19]. We are going to use this base for the Yukawa sector but now on we are going to omit the prime symbol. In this new base, it can be shown that in the scalar sector there are five scalar bosons,

$$
\phi^{ \pm}=H^{ \pm}, \quad \lambda_{2}^{0}=A^{0}, \phi_{1}^{0}, \phi_{2}^{0},
$$

where $\phi_{1}^{0}, \phi_{2}^{0}$ rotate and in the mass eigenstates they are

$$
\left(\begin{array}{c}
h^{0} \\
H^{0}
\end{array}\right)=\left(\begin{array}{cc}
\cos \alpha & \sin \alpha \\
-\sin \alpha & \cos \alpha
\end{array}\right)\left(\begin{array}{l}
\phi_{1}^{0} \\
\phi_{2}^{0}
\end{array}\right) \text {. }
$$


Where the parameter $\alpha$ can be taken in the range $-\frac{\pi}{2} \leqslant \alpha \leqslant \frac{\pi}{2}[20]$. Thus, from the scalar potential arises up five Higgs boson mass eigenstates, they are $H^{0}, h^{0}, H^{ \pm}$and $A^{0}$.

From the Lagrangian's terms of expression (11), we get that the mass matrices for fermions are given by,

$$
M^{f}=\frac{v}{\sqrt{2}} \eta^{f, 0} .
$$

And the Hermitian mass matrix $\mathrm{M}$ is diagonalized by a rotation matrix, according to

$$
V^{\dagger} M V=O^{T} P^{\dagger} M P O=O^{T} \widetilde{M} O=\left(\begin{array}{ccc} 
\pm m_{1} & 0 & 0 \\
0 & \mp m_{2} & 0 \\
0 & 0 & m_{3}
\end{array}\right)
$$

where $\widetilde{M}=P^{\dagger} M P$ is a real symmetric mass matrix, $O$ is an orthogonal matrix, $P=\operatorname{diag}\left(1, e^{-i \psi}, e^{-i(\psi+\theta)}\right)$ and $m_{1}, m_{2}, m_{3}$ correspond to the fermion masses. The upper signs are associated to the Fritzsch and Du-Xing ansatze, while the lower ones are used for the Fukuyama-Nishiura ansatz (See section III).

The fermion mass eigenstates are related to the interaction eigenstates by bi-unitary transformations [19],

$$
U_{L}^{0}=V_{L}^{U} U_{L}, \quad U_{R}^{0}=V_{R}^{U} U_{R}, \quad D_{L}^{0}=V_{L}^{D} D_{L}, \quad D_{R}^{0}=V_{R}^{D} D_{R}
$$

and therefore the CKM matrix will be defined as

$$
K=\left(V_{L}^{U}\right)^{\dagger} V_{L}^{D}=O_{U}^{T} P_{U D} O_{D}, \quad \text { where } \quad P_{U D}=P_{U}^{\dagger} P_{D}=\operatorname{diag}\left(1, e^{i \sigma}, e^{i \tau}\right)
$$

being $\sigma=\psi_{U}-\psi_{D}$ and $\tau=\psi_{U}-\psi_{D}+\theta_{U}-\theta_{D} . \psi_{U, D}$ and $\theta_{U, D}$ are the phases of the mass matrices for the upand down-type quarks, respectively. Further, when the transition to the mass eigenstates is performed in the Yukawa Lagrangian (1), the following relationships corresponding to the "rotated" coupling matrices are gotten [19],

$$
M_{U}^{\text {diag }}=\frac{v}{\sqrt{2}} V_{L}^{U \dagger} \eta^{U, 0} V_{R}^{U} \quad M_{D}^{\text {diag }}=\frac{v}{\sqrt{2}} V_{L}^{D \dagger} \eta^{D, 0} V_{R}^{D} \quad \xi^{U}=V_{L}^{U \dagger} \xi^{U, 0} V_{R}^{U}, \quad \xi^{D}=V_{L}^{D \dagger} \xi^{D, 0} V_{R}^{D} .
$$

Then eliminating $\eta^{f, 0}$ and $\xi^{f, 0}$ using equation (11), the Yukawa Lagrangian corresponding to the quark sector for the two Higgs Doublet Model type III takes the following form [19, 20],

$$
\begin{aligned}
-L_{Y} & =\bar{U} M_{U}^{\text {diag } U}+\bar{D} M_{D}^{\text {diag }} D+\frac{1}{v} \bar{U} M_{U}^{\text {diag }} U\left(\cos \alpha H^{0}-\sin \alpha h^{0}\right)+\frac{1}{\sqrt{2}} \bar{U}\left(\xi^{U} P_{R}+\left(\xi^{U}\right)^{\dagger} P_{L}\right) U\left(\sin \alpha H^{0}+\cos \alpha h^{0}\right) \\
& +\frac{1}{v} \bar{D} M_{D}^{\text {diag }} D\left(\cos \alpha H^{0}-\sin \alpha h^{0}\right)+\frac{1}{\sqrt{2}} \bar{D}\left(\xi^{D} P_{R}+\left(\xi^{D}\right)^{\dagger} P_{L}\right) D\left(\sin \alpha H^{0}+\cos \alpha h^{0}\right)-\frac{i}{v} \bar{D} M_{D}^{\text {diag }} \gamma_{5} D G_{Z}^{0} \\
& +\frac{i}{v} \bar{U} M_{U}^{\text {diag }} \gamma_{5} U G_{Z}^{0}-\frac{i}{\sqrt{2}} \bar{U}\left(\xi^{U} P_{R}-\left(\xi^{U}\right)^{\dagger} P_{L}\right) U A^{0}+\frac{i}{\sqrt{2}} \bar{D}\left(\xi^{D} P_{R}-\left(\xi^{D}\right)^{\dagger} P_{L}\right) D A^{0} \\
& +\frac{\sqrt{2}}{v} \bar{U}\left(K M_{D}^{\text {diag }} P_{R}-M_{U}^{\text {diag }} K P_{L}\right) D G_{W}^{+}-\frac{\sqrt{2}}{v} \bar{D}\left(K^{\dagger} M_{U}^{\text {diag }} P_{R}-M_{D}^{\text {diag }} K^{\dagger} P_{L}\right) U G_{W}^{-} \\
& +\bar{U}\left(K \xi^{D} P_{R}-\left(\xi^{U}\right)^{\dagger} K P_{L}\right) D H^{+}-\bar{D}\left(K^{\dagger} \xi^{U} P_{R}-\left(\xi^{D}\right)^{\dagger} K^{\dagger} P_{L}\right) U H^{-} .
\end{aligned}
$$

Notice that if $\xi^{U}$ and $\xi^{D}$ vanish from this Yukawa Lagrangian, the 2HDM type II is reproduced. The new terms are leading to flavor changing processes at tree level.

\section{DIFFERENT KIND OF ANSATZE}

In this section different mass matrices ansatze are presented. The Yukawa coupling matrices in the mass basis are obtained, assuming that the Yukawa coupling matrices have the same structure that the mass matrices. In the framework of the 2HDM type III the flavor changing couplings at tree level will depend on the ansatz parameters. The Fritzsch ansatz is the first one reviewed because is the simplest one and it was the motivation for the widely known and used Cheng-Sher ansatz in the 2HDM-III phenomenology. Then the Du-Xing ansatz is discussed and it is followed by combinations of the Fritzsch and the Du-Xing ansatze looking for the best fit of the CKM elements. Furthermore, the Fukuyama-Nishiura ansatz is explored and a new type of ansatz which is controlling the FCNC processes in the top quark sector is proposed. It is worth to mention that phases are explicitly involved since the mass matrices are hermitic, and then they can be written always as $M=P \tilde{M} P^{\dagger}$, where $\tilde{M}$ is a real and symmetric matrix and $P$ is a diagonal matrix which contains the phases [11]. 


\section{A. Fritzsch Ansatz (FA)}

For the three family case, a mass matrix ansatz proposed by Fritzsch has been widely discussed [1, 10, 14], it is

$$
M=\left(\begin{array}{ccc}
0 & D & 0 \\
D^{*} & 0 & B \\
0 & B^{*} & A
\end{array}\right)=\left(\begin{array}{ccc}
1 & 0 & 0 \\
0 & e^{-i \psi} & 0 \\
0 & 0 & e^{-i(\psi+\theta)}
\end{array}\right)\left(\begin{array}{ccc}
0 & |D| & 0 \\
|D| & 0 & |B| \\
0 & |B| & A
\end{array}\right)\left(\begin{array}{ccc}
1 & 0 & 0 \\
0 & e^{i \psi} & 0 \\
0 & 0 & e^{i(\psi+\theta)}
\end{array}\right)=P \widetilde{M} P^{\dagger}
$$

And motivated by the Fritzsch texture mass matrix, Cheng and Sher have proposed an ansatz for the Yukawa flavor changing coupling matrices in the interaction basis according to [10, 14]:

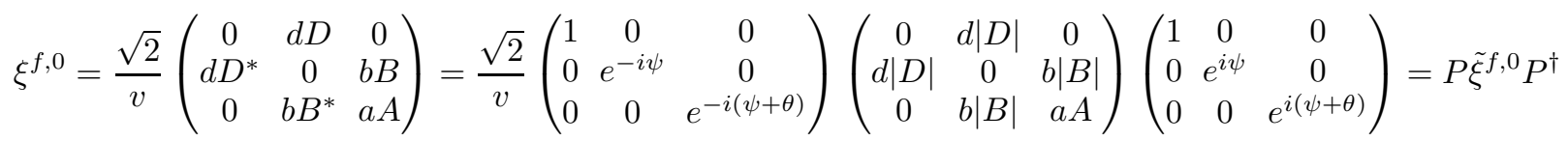

where $f=U, D$, the coefficients $a, b, d$ are of the order of one, and the parameters $A,|B|$ and $|D|$ are given by,

$$
\begin{gathered}
A=m_{3}-m_{2}+m_{1}, \quad|D|=\left(\frac{m_{1} m_{2} m_{3}}{m_{3}-m_{2}+m_{1}}\right)^{1 / 2}, \\
|B|=\left(m_{1} m_{2}+m_{3} m_{2}-m_{1} m_{3}-\frac{m_{1} m_{2} m_{3}}{m_{3}+m_{1}-m_{2}}\right)^{1 / 2} .
\end{gathered}
$$

But taking into account the mass hierarchy $m_{1}<<m_{2}<<m_{3}$, the expressions (15) reduce to

$$
A \approx m_{3}, \quad|B| \approx \sqrt{m_{2} m_{3}}, \quad|D| \approx \sqrt{m_{1} m_{2}}
$$

The rotation matrix which diagonalizes $\widetilde{M}$ is given by

$$
O \simeq\left(\begin{array}{ccc}
1 & -\sqrt{\frac{m_{1}}{m_{2}}} & 0 \\
\sqrt{\frac{m_{1}}{m_{2}}} & 1 & \sqrt{\frac{m_{2}}{m_{3}}} \\
-\sqrt{\frac{m_{1}}{m_{3}}} & -\sqrt{\frac{m_{2}}{m_{3}}} & 1
\end{array}\right)
$$

Then using the mass matrix (13) for up-type and down-type quarks and equations (10) and (17), the following expressions of the CKM matrix elements are gotten

$$
\begin{gathered}
K_{u d} \simeq 1+e^{i \sigma} \sqrt{\frac{m_{u} m_{d}}{m_{c} m_{s}}}+e^{i \tau} \sqrt{\frac{m_{u} m_{d}}{m_{t} m_{b}}}, \quad K_{u s} \simeq e^{i \sigma} \sqrt{\frac{m_{u}}{m_{c}}}-\sqrt{\frac{m_{d}}{m_{s}}}+e^{i \tau} \sqrt{\frac{m_{u} m_{s}}{m_{t} m_{b}}} \\
K_{u b} \simeq \sqrt{\frac{m_{u}}{m_{c}}}\left(e^{i \sigma} \sqrt{\frac{m_{s}}{m_{b}}}-e^{i \tau} \sqrt{\frac{m_{c}}{m_{t}}}\right), \quad K_{c d} \simeq e^{i \sigma \sqrt{\frac{m_{d}}{m_{s}}}}-\sqrt{\frac{m_{u}}{m_{c}}}+e^{i \tau} \sqrt{\frac{m_{c} m_{d}}{m_{t} m_{b}}} \\
K_{c s} \simeq e^{i \sigma}+\sqrt{\frac{m_{u} m_{d}}{m_{c} m_{s}}}+e^{i \tau} \sqrt{\frac{m_{c} m_{s}}{m_{t} m_{b}}}, \quad K_{c b} \simeq e^{i \sigma} \sqrt{\frac{m_{s}}{m_{b}}}-e^{i \tau} \sqrt{\frac{m_{c}}{m_{t}}}, \\
K_{t d} \simeq \sqrt{\frac{m_{d}}{m_{s}}}\left(e^{i \sigma} \sqrt{\frac{m_{c}}{m_{t}}}-e^{i \tau} \sqrt{\frac{m_{s}}{m_{b}}}\right), \quad K_{t s} \simeq e^{i \sigma \sqrt{\frac{m_{c}}{m_{t}}}}-e^{i \tau} \sqrt{\frac{m_{s}}{m_{b}}}, \quad K_{t b} \simeq e^{i \tau}+e^{i \sigma} \sqrt{\frac{m_{c} m_{s}}{m_{t} m_{b}}} .
\end{gathered}
$$

In order to get the best agreement of the $\left|K_{u s}\right|$ and $\left|K_{c d}\right|$ elements with their experimental values, we use $\sigma=81.57^{\circ}$ and $\tau=-11.78^{\circ}$. Hence $\psi_{U}=\psi_{D}+81.57^{\circ}$ and $\theta_{U}=\theta_{D}-93.35^{\circ}$. The numerical values shown in table $\llbracket$ for the CKM matrix elements are obtained using the mass values listed in appendix A. From these results, a considerable 
disagreement of the $\left|K_{u b}\right|,\left|K_{c b}\right|,\left|K_{t d}\right|$ and $\left|K_{t s}\right|$ elements with their experimental values is noticed. On the other hand, the $\mathrm{CP}$ violating phase $\delta$ for the Fritzsch ansatz is obtained, $\delta=86.61^{\circ}$ by using the expression [11]:

$$
\sin \delta=\frac{\left(1-\left|K_{u b}\right|^{2}\right) J}{\left|K_{u d} K_{u s} K_{u b} K_{c b} K_{t b}\right|},
$$

where $J$ is the Jarlskog invariant given by $J=\Im m\left(K_{u s} K_{c b} K_{u b}^{*} K_{c s}^{*}\right)$ [11, 12]. This CP phase $\delta$ presents a discrepancy of about $30.54 \%$ with respect to the experimental value $\delta=60.16^{\circ} \pm 14^{\circ}$.

On other hand, the Yukawa coupling matrices in the mass basis are given by [10]

$$
\widetilde{\xi}^{U, D}=O_{U, D}^{T} \widetilde{\widetilde{\xi}}^{U, D, 0} O_{U, D}
$$

where $\widetilde{\xi}^{U, D, 0}=P^{\dagger} \xi^{U, D, 0} P$. Therefore, taking into account the mass hierarchy and using the expression $\xi^{U, D}=P O_{U, D}^{T} \tilde{\xi}^{U, D, 0} O_{U, D} P^{\dagger}$, the Yukawa coupling matrices in the mass basis are given by

$$
\begin{aligned}
& \xi^{U} \simeq \frac{\sqrt{2}}{v}\left(\begin{array}{ccc}
\left(2 d^{(U)}-2 b^{(U)}+a^{(U)}\right) m_{u} & \left.a^{(U)}+d^{(U)}-2 b^{(U)}\right) \sqrt{m_{u} m_{c}} e^{i \psi_{U}} & \left(b^{(U)}-a^{(U)}\right) \sqrt{m_{u} m_{t}}{ }^{i\left(\psi_{U}+\theta_{U}\right)} \\
\left(a^{(U)}+d^{(U)}-2 b^{(U)}\right) \sqrt{m_{u} m_{c}} e^{-i \psi_{U}} & \left(a^{(U)}-2 b^{(U)}\right) m_{c} & \left(b^{(U)}-a^{(U)}\right) \sqrt{m_{c} m_{t}} e^{i \theta_{U}} \\
\left(b^{(U)}-a^{(U)}\right) \sqrt{m_{u} m_{t}} e^{-i\left(\psi_{U}+\theta_{U}\right)} & \left(b^{(U)}-a^{(U)}\right) \sqrt{m_{c} m_{t}} e^{-i \theta_{U}} & a^{(U)} m_{t}
\end{array}\right), \\
& \xi^{D} \simeq \frac{\sqrt{2}}{v}\left(\begin{array}{ccc}
\left(2 d^{(D)}-2 b^{(D)}+a^{(D)}\right) m_{d} & \left(a^{(D)}+d^{(D)}-2 b^{(D)}\right) \sqrt{m_{d} m_{s}} e^{i \psi_{D}} & \left(b^{(D)}-a^{(D)}\right) \sqrt{m_{d} m_{b}}{ }^{i\left(\psi_{D}+\theta_{D}\right)} \\
\left(a^{(D)}+d^{(D)}-2 b^{(D)}\right) \sqrt{m_{d} m_{s}} e^{-i \psi_{D}} & \left(a^{(D)}-2 b^{(D)}\right) m_{s} & \left(b^{(D)}-a^{(D)}\right) \sqrt{m_{s} m_{b}} e^{i \theta_{D}} \\
\left(b^{(D)}-a^{(D)}\right) \sqrt{m_{d} m_{b}} e^{-i\left(\psi_{D}+\theta_{D}\right)} & \left(b^{(D)}-a^{(D)}\right) \sqrt{m_{s} m_{b}} e^{-i \theta_{D}} & a^{(D)} m_{b}
\end{array}\right),
\end{aligned}
$$

equation (21) reproduces the expression obtained by Cheng and Sher [10] when the complex phases vanish. Further, we can see that the hierarchy structure of the Yukawa coupling matrices in the mass basis are dominated by the fermion masses and the $\left(\xi^{U, D}\right)_{i j}$ elements are proportional to $\sqrt{m_{i} m_{j}}$, which is the usual Cheng-Sher ansatz [10].

\begin{tabular}{|c|c|c|c|c|c|c|c|}
\hline & FA & DXA & DXFA & FDXA & $\tilde{X}$ A & FNA & NTA \\
\hline$K_{u d}$ & 1.00189 & 1.00189 & 1.00189 & 1.00189 & 1.00189 & 0.97467 & 1.00051 \\
\hline$K_{u s}$ & 0.22720 & 0.22720 & 0.22720 & 0.22720 & 0.22720 & 0.22361 & 0.22720 \\
\hline$K_{u b}$ & 0.00797 & 0.00090 & 0.00734 & 0.00359 & 0.00181 & 0.00308 & 0.00190 \\
\hline$K_{c d}$ & 0.22710 & 0.22721 & 0.22710 & 0.22710 & 0.22718 & 0.22341 & 0.22720 \\
\hline$K_{c s}$ & 1.00144 & 1.00194 & 1.00146 & 1.00144 & 1.00178 & 0.97381 & 1.00051 \\
\hline$K_{c b}$ & 0.15512 & 0.01746 & 0.14277 & 0.06986 & 0.03562 & 0.04221 & 0.03197 \\
\hline$K_{t d}$ & 0.03559 & 0.00401 & 0.03275 & 0.01603 & 0.00817 & 0.01002 & 0.00735 \\
\hline$K_{t s}$ & 0.15512 & 0.01746 & 0.14277 & 0.06986 & 0.03562 & 0.04112 & 0.03203 \\
\hline$K_{t b}$ & 0.99955 & 1.00004 & 0.99956 & 0.99955 & 0.99988 & 0.99910 & 1 \\
\hline$\delta$ & $86.61^{\circ}$ & $86.66^{\circ}$ & $86.62^{\circ}$ & $86.66^{\circ}$ & $82.18^{\circ}$ & $-87.55^{\circ}$ & $80.68^{\circ}$ \\
\hline \hline
\end{tabular}

TABLE I: The CKM matrix elements and CP violating phase $\delta$ for the different ansatze considered.

\section{B. Du-Xing Ansatz (DXA)}

In order to accommodate all the current data of quark masses and mixing angles in the framework of texture-zeros, the following ansatz for the mass matrix was suggested [1, 10, 14],

$$
M=\left(\begin{array}{ccc}
0 & D & 0 \\
D^{*} & C & B \\
0 & B^{*} & A
\end{array}\right)=P \widetilde{M} P^{\dagger} \quad \text { with } \quad|A| \gg|B|,|C| \gg|D|
$$




\begin{tabular}{|c|c|c|c|c|c|c|c|}
\hline & FA & DXA & DXFA & FDXA & $X$ A & FNA & NTA \\
\hline$K_{u d}$ & 2.80 & 2.80 & 2.80 & 2.80 & 2.80 & 0.09 & 2.67 \\
\hline$K_{u s}$ & 0.00 & 0.00 & 0.00 & 0.00 & 0.00 & 1.60 & 0.00 \\
\hline$K_{u b}$ & 50.32 & 341.21 & 46.03 & 10.30 & 118.31 & 28.72 & 108.64 \\
\hline$K_{c d}$ & 0.00 & 0.05 & 0.00 & 0.00 & 0.03 & 1.65 & 0.04 \\
\hline$K_{c s}$ & 2.84 & 2.89 & 2.84 & 2.84 & 2.88 & 0.09 & 2.75 \\
\hline$K_{c b}$ & 72.79 & 141.69 & 70.44 & 38.58 & 18.50 & 0.00 & 32.01 \\
\hline$K_{t d}$ & 77.13 & 103.16 & 75.15 & 49.21 & 0.39 & 18.80 & 10.77 \\
\hline$K_{t s}$ & 73.18 & 138.25 & 70.86 & 40.44 & 16.81 & 1.20 & 29.91 \\
\hline$K_{t b}$ & 0.04 & 0.09 & 0.05 & 0.04 & 0.08 & 0.00 & 0.09 \\
\hline$\delta$ & 30.54 & 30.58 & 30.55 & 30.58 & 26.79 & 168.72 & 25.43 \\
\hline \hline
\end{tabular}

TABLE II: Error percentages of the CKM matrix elements and CP violating phase $\delta$ for the different ansatze considered. The experimental values used are those reported by [21]

where $A \simeq m_{3},|B| \simeq|C| \simeq m_{2}$, and $|D| \simeq \sqrt{m_{1} m_{2}}$. The rotation matrix for this case is [10]:

$$
O \simeq\left(\begin{array}{ccc}
1 & \sqrt{\frac{m_{1}}{m_{2}}} & 0 \\
-\sqrt{\frac{m_{1}}{m_{2}}} & 1 & \frac{m_{2}}{m_{3}} \\
\frac{\sqrt{m_{1} m_{2}}}{m_{3}} & -\frac{m_{2}}{m_{3}} & 1
\end{array}\right) .
$$

And the CKM matrix elements obtained from the mass matrix ansatz given in (22) are 14]

$$
\begin{gathered}
K_{u d} \simeq 1+e^{i \sigma} \sqrt{\frac{m_{u} m_{d}}{m_{c} m_{s}}}+e^{i \tau} \frac{\sqrt{m_{u} m_{c} m_{d} m_{s}}}{m_{t} m_{b}}, \quad K_{u s} \simeq \sqrt{\frac{m_{d}}{m_{s}}}-e^{i \sigma} \sqrt{\frac{m_{u}}{m_{c}}}-e^{i \tau} \frac{m_{s} \sqrt{m_{u} m_{c}}}{m_{t} m_{b}} \\
K_{u b} \simeq \sqrt{\frac{m_{u}}{m_{c}}}\left(\frac{m_{c}}{m_{t}} e^{i \tau}-\frac{m_{s}}{m_{b}} e^{i \sigma}\right), \quad K_{c d} \simeq \sqrt{\frac{m_{u}}{m_{c}}}-e^{i \sigma} \sqrt{\frac{m_{d}}{m_{s}}}-e^{i \tau} \frac{m_{c} \sqrt{m_{d} m_{s}}}{m_{t} m_{b}}, \\
K_{c s} \simeq e^{i \sigma}+\sqrt{\frac{m_{u} m_{d}}{m_{c} m_{s}}}+\frac{m_{c} m_{s}}{m_{t} m_{b}} e^{i \tau}, \quad K_{c b} \simeq \frac{m_{s}}{m_{b}} e^{i \sigma}-\frac{m_{c}}{m_{t}} e^{i \tau}, \\
K_{t d} \simeq \sqrt{\frac{m_{d}}{m_{s}}}\left(\frac{m_{s}}{m_{b}} e^{i \tau}-\frac{m_{c}}{m_{t}} e^{i \sigma}\right), \quad K_{t s} \simeq \frac{m_{c}}{m_{t}} e^{i \sigma}-\frac{m_{s}}{m_{b}} e^{i \tau}, \quad K_{t b} \simeq e^{i \tau}+\frac{m_{c} m_{s}}{m_{t} m_{b}} e^{i \sigma} .
\end{gathered}
$$

The best agreement of the $\left|K_{u s}\right|$ and $\left|K_{c d}\right|$ elements with their experimental values is gotten when $\sigma=81.09^{\circ}$ and $\tau=29.97^{\circ}$. Then $\psi_{U}=\psi_{D}+81.09^{\circ}$ and $\theta_{U}=\theta_{D}-51.12^{\circ}$. The numerical values of the CKM matrix elements are shown in table \. There are a considerable disagreement between the magnitudes of the $\left|K_{u b}\right|,\left|K_{c b}\right|,\left|K_{t d}\right|$ and $\left|K_{t s}\right|$ elements and their experimental values. On the other hand, the obtained CP violating phase for the Du-Xing ansatz is $\delta=86.66^{\circ}$. This result has an inconsistency with the experimental value [21] of about $30.58 \%$.

The table $\amalg$ shows the error percentages of the CKM matrix elements and CP violating phase $\delta$ for the different ansatze. The deviations of the CKM matrix elements obtained from the Du-Xing ansatz are higher than the corresponding to the Fritzsch ansatz. Then, the Fritzsch ansatz leads to a better prediction of the CKM matrix elements than the Du-Xing ansatz. About the CP violating phase, the Fritzsch ansatz leads to approximately the same prediction of this phase than the obtained from the Du-Xing ansatz.

On other hand, the Yukawa coupling matrices in the mass basis are

$$
\xi^{U} \simeq \frac{\sqrt{2}}{v}\left(\begin{array}{ccc}
\left(c^{(U)}-2 d^{(U)}\right) m_{u} & \left(d^{(U)}-c^{(U)}\right) \sqrt{m_{u} m_{c}} e^{i \psi_{U}} & \left(a^{(U)}-b^{(U)}\right) \sqrt{m_{u} m_{c}} e^{i\left(\psi_{U}+\theta_{U}\right)} \\
\left(d^{(U)}-c^{(U)}\right) \sqrt{m_{u} m_{c}} e^{-i \psi_{U}} & c^{(U)} m_{c} & \left(b^{(U)}-a^{(U)}\right) m_{c} e^{i \theta_{U}} \\
\left(a^{(U)}-b^{(U)}\right) \sqrt{m_{u} m_{c}} e^{-i\left(\psi_{U}+\theta_{U}\right)} & \left(b^{(U)}-a^{(U)}\right) m_{c} e^{-i \theta_{U}} & a^{(U)} m_{t}
\end{array}\right)
$$




$$
\xi^{D} \simeq \frac{\sqrt{2}}{v}\left(\begin{array}{ccc}
\left(c^{(D)}-2 d^{(D)}\right) m_{d} & \left(d^{(D)}-c^{(D)}\right) \sqrt{m_{d} m_{s}} e^{i \psi_{D}} & \left(a^{(D)}-b^{(D)}\right) \sqrt{m_{d} m_{s}} e^{i\left(\psi_{D}+\theta_{D}\right)} \\
\left(d^{(D)}-c^{(D)}\right) \sqrt{m_{d} m_{s}} e^{-i \psi_{D}} & c^{(D)} m_{s} & \left(b^{(D)}-a^{(D)}\right) m_{s} e^{i \theta_{D}} \\
\left(a^{(D)}-b^{(D)}\right) \sqrt{m_{d} m_{s}} e^{-i\left(\psi_{D}+\theta_{D}\right)} & \left(b^{(D)}-a^{(D)}\right) m_{s} e^{-i \theta_{U}} & a^{(D)} m_{b}
\end{array}\right)
$$

using the Du-Xing ansatz (22) according to $\xi^{U, D}=P \widetilde{\sim}^{U, D} P^{\dagger}$. Then, the Du-Xing ansatz lead to the same structure of the Yukawa couplings for the first and the second generation fermions. And the coupling to the third generation fermions in the expressions (25) and (26) are weaker than the predicted by the Fritzsch ansatz.

\section{Combination of the Fritzsch and the Du-Xing ansatz}

\section{The Du-Xing ansatz for the up sector and the Fritzsch ansatz for the down sector (DFXA)}

By using the Fritzsch and the Du-Xing ansatze for the down- and up-type quarks respectively, the following expressions corresponding to the CKM matrix elements are obtained,

$$
\begin{aligned}
& K_{u d} \simeq 1-e^{i \sigma} \sqrt{\frac{m_{d} m_{u}}{m_{s} m_{c}}}-e^{i \tau} \frac{\sqrt{m_{u} m_{c} m_{d}}}{m_{t} \sqrt{m_{b}}}, \quad K_{u s} \simeq-\sqrt{\frac{m_{d}}{m_{s}}}-e^{i \sigma} \sqrt{\frac{m_{u}}{m_{c}}}-e^{i \tau} \frac{\sqrt{m_{u} m_{c} m_{s}}}{m_{t} \sqrt{m_{b}}} \\
& K_{u b} \simeq e^{i \tau} \frac{\sqrt{m_{u} m_{c}}}{m_{t}}-e^{i \sigma} \sqrt{\frac{m_{u} m_{s}}{m_{c} m_{b}}}, \quad K_{c d} \simeq e^{i \sigma} \sqrt{\frac{m_{d}}{m_{s}}}+\sqrt{\frac{m_{u}}{m_{c}}}+e^{i \tau} \frac{m_{c}}{m_{t}} \sqrt{\frac{m_{d}}{m_{b}}}, \\
& K_{c s} \simeq e^{i \sigma}+e^{i \tau} \frac{m_{c}}{m_{t}} \sqrt{\frac{m_{s}}{m_{b}}}-\sqrt{\frac{m_{d} m_{u}}{m_{s} m_{c}}}, \quad K_{c b} \simeq e^{i \sigma} \sqrt{\frac{m_{s}}{m_{b}}}-e^{i \tau} \frac{m_{c}}{m_{t}} \\
& K_{t d} \simeq e^{i \sigma} \frac{m_{c}}{m_{t}} \sqrt{\frac{m_{d}}{m_{s}}}-e^{i \tau} \sqrt{\frac{m_{d}}{m_{b}}}, \quad K_{t s} \simeq e^{i \sigma} \frac{m_{c}}{m_{t}}-e^{i \tau} \sqrt{\frac{m_{s}}{m_{b}}}, \quad K_{t b} \simeq e^{i \tau}+e^{i \sigma} \frac{m_{c}}{m_{t}} \sqrt{\frac{m_{s}}{m_{b}}}
\end{aligned}
$$

With the aim to get a better agreement of the $\left|K_{u d}\right|$ and $\left|K_{c d}\right|$ elements with their experimental values we obtain $\sigma=81.09^{\circ}$ and $\tau=29.97^{\circ}$, and then $\psi_{U}=\psi_{D}+90^{\circ}$ and $\theta_{U}=\theta_{D}-51.12^{\circ}$. The numerical values of the CKM matrix elements for this case are shown in Table I In this case, there is good agreement of the CKM matrix elements with their experimental values, with the exception of the $\left|K_{u b}\right|,\left|K_{c b}\right|,\left|K_{t d}\right|$ and $\left|K_{t s}\right|$ elements, which present discrepancies of about $46.03 \%, 70.44 \%, 75.15 \%$ and $70.86 \%$, respect to their corresponding experimental values. On the other hand, we obtain that the $\mathrm{CP}$ violating phase is $\delta=86.62^{\circ}$. This result presents a discrepancy of about $30.55 \%$.

About the Yukawa coupling matrices in the mass basis for this case, they are

$$
\begin{gathered}
\xi^{U} \simeq \frac{\sqrt{2}}{v}\left(\begin{array}{ccc}
\left(c^{(U)}-2 d^{(U)}\right) m_{u} & \left(d^{(U)}-c^{(U)}\right) \sqrt{m_{u} m_{c}} e^{i \psi_{U}} & \left(a^{(U)}-b^{(U)}\right) \sqrt{m_{u} m_{c}} e^{i\left(\psi_{U}+\theta_{U}\right)} \\
\left(d^{(U)}-c^{(U)}\right) \sqrt{m_{u} m_{c}} e^{-i \psi_{U}} & c^{(U)} m_{c} & \left(b^{(U)}-a^{(U)}\right) m_{c} e^{i \theta_{U}} \\
\left(a^{(U)}-b^{(U)}\right) \sqrt{m_{u} m_{c}} e^{-i\left(\psi_{U}+\theta_{U}\right)} & \left(b^{(U)}-a^{(U)}\right) m_{c} e^{-i \theta_{U}} & a^{(U)} m_{t}
\end{array}\right) \\
\xi^{D} \simeq \frac{\sqrt{2}}{v}\left(\begin{array}{ccc}
(28) \\
\left(a^{(D)}+d^{(D)}-2 b^{(D)}+a^{(D)}\right) m_{d} & \left.\left(a^{(D)}+d^{(D)}\right) \sqrt{m_{d} m_{s}} e^{-i \psi_{D}}-2 b^{(D)}\right) \sqrt{m_{d} m_{s}} e^{i \psi_{D}} & \left(b^{(D)}-a^{(D)}\right) \sqrt{m_{d} m_{b}} \\
\left(b^{(D)}-a^{(D)}\right) \sqrt{m_{d} m_{b}} e^{-i\left(\psi_{D}+\theta_{D}\right)} & \left(a^{(D)}-2 b^{(D)}\right) m_{s} & \left(b^{(D)}-a^{(D)}\right) \sqrt{m_{s} m_{b}} e^{i \theta_{D}}
\end{array}\right) .
\end{gathered}
$$

\section{The Fritzsch ansatz for the up quark sector and the Du-Xing ansatz for the down quark sector (FDXA)}

In this section we use the Fritzsch and the Du-Xing ansatze for the up-type and down-type quarks respectively. It is contrary to the DXFA case discussed before. The expressions for the CKM matrix elements are: 


$$
\begin{gathered}
K_{u d} \simeq 1-e^{i \sigma} \sqrt{\frac{m_{d} m_{u}}{m_{s} m_{c}}}-e^{i \tau} \frac{\sqrt{m_{u} m_{d} m_{s}}}{m_{b} \sqrt{m_{t}}}, \quad K_{u s} \simeq \sqrt{\frac{m_{d}}{m_{s}}}+e^{i \sigma} \sqrt{\frac{m_{u}}{m_{c}}}+e^{i \tau} \frac{m_{s}}{m_{b}} \sqrt{\frac{m_{u}}{m_{t}}} \\
K_{u b} \simeq e^{i \sigma} \frac{m_{s}}{m_{b}} \sqrt{\frac{m_{u}}{m_{c}}}-e^{i \tau} \sqrt{\frac{m_{u}}{m_{t}}}, \quad K_{c d} \simeq-e^{i \sigma} \sqrt{\frac{m_{d}}{m_{s}}}-\sqrt{\frac{m_{u}}{m_{c}}}-e^{i \tau} \frac{\sqrt{m_{c} m_{d} m_{s}}}{m_{b} \sqrt{m_{t}}} \\
K_{c s} \simeq e^{i \sigma}+e^{i \tau} \frac{m_{s}}{m_{b}} \sqrt{\frac{m_{c}}{m_{t}}}-\sqrt{\frac{m_{d} m_{u}}{m_{s} m_{c}}}, \quad K_{c b} \simeq e^{i \sigma \frac{m_{s}}{m_{b}}}-e^{i \tau} \sqrt{\frac{m_{c}}{m_{t}}}, \\
K_{t d} \simeq e^{i \tau} \frac{\sqrt{m_{d} m_{s}}}{m_{b}}-e^{i \sigma} \sqrt{\frac{m_{d} m_{c}}{m_{s} m_{t}}}, \quad K_{t s} \simeq e^{i \sigma} \sqrt{\frac{m_{c}}{m_{t}}}-e^{i \tau} \frac{m_{s}}{m_{b}}, \quad e^{i \tau}+e^{i \sigma} \frac{m_{s}}{m_{b}} \sqrt{\frac{m_{c}}{m_{t}}} .
\end{gathered}
$$

In order to get the best agreement of the $\left|K_{u s}\right|$ and $\left|K_{c d}\right|$ elements with their experimental values, we get $\sigma=-98.93^{\circ}$ and $\tau=52.19^{\circ}$. And $\psi_{U}=\psi_{D}-98.93^{\circ}$ and $\theta_{U}=\theta_{D}+151.12^{\circ}$ are found. Then according to the previous results, the numerical values are presented in Table I In this case, there is good agreement of the CKM matrix elements with their experimental values, with the exception of the $\left|K_{c b}\right|,\left|K_{t d}\right|,\left|K_{t s}\right|$ elements, which present discrepancies of about $38.58 \%, 49.21 \%$, and $40.44 \%$, respect to their corresponding experimental values. On the other hand, we obtain that the $\mathrm{CP}$ violating phase for this case is $\delta=86.66^{\circ}$. This result is deviated $30.58 \%$ from the experimental value.

In the Table $\llbracket$ the error percentages of the CKM matrix elements are lower than the corresponding to the $\left|K_{i j}\right|$ elements given by the DFXA case. This is an indication that when the prescription FDXA is used, we obtain a better agreement with the experimental values of the CKM matrix elements than in the combinations considered before.

On the other hand, the following Yukawa coupling matrices in the mass basis are obtained,

$$
\begin{aligned}
& \xi^{U} \simeq \frac{\sqrt{2}}{v}\left(\begin{array}{ccc}
\left(2 d^{(U)}-2 b^{(U)}+a^{(U)}\right) m_{u} & \left(a^{(U)}+d^{(U)}-2 b^{(U)}\right) \sqrt{m_{u} m_{c}} e^{i \psi_{U}} & \left(b^{(U)}-a^{(U)}\right) \sqrt{m_{u} m_{t}}\left(\psi_{U}+\theta_{U}\right) \\
\left(a^{(U)}+d^{(U)}-2 b^{(U)}\right) \sqrt{m_{u} m_{c}} e^{-i \psi_{U}} & \left(a^{(U)}-2 b^{(U)}\right) m_{c} & \left(b^{(U)}-a^{(U)}\right) \sqrt{m_{c} m_{t}} e^{i \theta_{U}} \\
\left(b^{(U)}-a^{(U)}\right) \sqrt{m_{u} m_{t}} e^{-i\left(\psi_{U}+\theta_{U}\right)} & \left(b^{(U)}-a^{(U)}\right) \sqrt{m_{c} m_{t}} e^{-i \theta_{U}} & a^{(U)} m_{t}
\end{array}\right) \\
& \xi^{D} \simeq \frac{\sqrt{2}}{v}\left(\begin{array}{ccc}
\left(c^{(D)}-2 d^{(D)}\right) m_{d} & \left(d^{(D)}-c^{(D)}\right) \sqrt{m_{d} m_{s}} e^{i \psi_{D}} & \left(a^{(D)}-b^{(D)}\right) \sqrt{m_{d} m_{s}} e^{i\left(\psi_{D}+\theta_{D}\right)} \\
\left(d^{(D)}-c^{(D)}\right) \sqrt{m_{d} m_{s}} e^{-i \psi_{D}} & c^{(D)} m_{s} & \left(b^{(D)}-a^{(D)}\right) m_{s} e^{i \theta_{D}} \\
\left(a^{(D)}-b^{(D)}\right) \sqrt{m_{d} m_{s}} e^{-i\left(\psi_{D}+\theta_{D}\right)} & \left(b^{(D)}-a^{(D)}\right) m_{s} e^{-i \theta_{U}} & a^{(D)} m_{b}
\end{array}\right)
\end{aligned}
$$

\section{Combination of different assignments in the Du-Xing ansatz $((\tilde{X} A)$}

It is known that taking $M^{\text {diag }}=\operatorname{diag}\left(-m_{1}, m_{2}, m_{3}\right)$, the following relations between the components of the $\widetilde{M}$ matrix given in (22) are satisfied [14],

$$
C+A=-m_{1}+m_{2}+m_{3}, \quad C A-|B|^{2}-|D|^{2}=-m_{1} m_{2}+m_{2} m_{3}+m_{3} m_{1}, \quad A|D|^{2}=m_{1} m_{2} m_{3} .
$$

The assumptions $A \gg|B|$ and $|C| \gg|D|$ define the assignment type $\mathrm{A}$ and the assumption $C=m_{2}$ define the assignment type B. For the assignment type B, the parameters are

$$
A=m_{3}-m_{1}, \quad|D|=\sqrt{\frac{m_{1} m_{2} m_{3}}{m_{3}-m_{1}}}, \quad|B|=\sqrt{\frac{m_{1} m_{3}\left(m_{3}-m_{2}-m_{1}\right)}{m_{3}-m_{1}}},
$$

and the rotation matrix $O$ is $[9]$ :

$$
O \simeq\left(\begin{array}{ccc}
1 & \sqrt{\frac{m_{1}}{m_{2}}} & \sqrt{\frac{m_{2} m_{1}^{2}}{m_{3}^{3}}} \\
-\sqrt{\frac{m_{1}}{m_{2}}} & 1 & \sqrt{\frac{m_{1}}{m_{3}}} \\
\sqrt{\frac{m_{1}^{2}}{m_{2} m_{3}}} & -\sqrt{\frac{m_{1}}{m_{3}}} & 1
\end{array}\right) \quad \text { for } \quad m_{3} \gg m_{2} \gg m_{1} .
$$


Then, the following expressions for the CKM matrix elements are obtained,

$$
\begin{aligned}
& K_{u d} \simeq 1+e^{i \sigma} \sqrt{\frac{m_{u} m_{d}}{m_{c} m_{s}}}+e^{i \tau} \frac{m_{d}}{m_{t}} \sqrt{\frac{m_{u} m_{c}}{m_{s} m_{b}}}, \quad K_{u s} \simeq \sqrt{\frac{m_{d}}{m_{s}}}-e^{i \sigma} \sqrt{\frac{m_{u}}{m_{c}}}-e^{i \tau} \frac{\sqrt{m_{u} m_{c} m_{d}}}{m_{t} \sqrt{m_{b}}} \\
& K_{u b} \simeq \sqrt{\frac{m_{s} m_{d}^{2}}{m_{b}^{3}}}-e^{i \sigma} \sqrt{\frac{m_{u} m_{d}}{m_{c} m_{b}}}+e^{i \tau} \frac{\sqrt{m_{u} m_{c}}}{m_{t}}, \quad K_{c d} \simeq \sqrt{\frac{m_{u}}{m_{c}}}-\frac{m_{c}}{m_{t}} \sqrt{\frac{m_{d}^{2}}{m_{s} m_{b}}} e^{i \tau}-e^{i \sigma} \sqrt{\frac{m_{d}}{m_{s}}}, \\
& K_{c s} \simeq e^{i \sigma}+\sqrt{\frac{m_{u} m_{d}}{m_{c} m_{s}}}+e^{i \tau} \frac{m_{c}}{m_{t}} \sqrt{\frac{m_{d}}{m_{b}}}, \quad K_{c b} \simeq e^{i \sigma} \sqrt{\frac{m_{d}}{m_{b}}}-\frac{m_{c}}{m_{t}} e^{i \tau}+\sqrt{\frac{m_{u} m_{s} m_{d}^{2}}{m_{c} m_{b}^{3}}}, \\
& K_{t d} \simeq e^{i \tau} \sqrt{\frac{m_{d}^{2}}{m_{s} m_{b}}}-e^{i \sigma} \frac{m_{c}}{m_{t}} \sqrt{\frac{m_{d}}{m_{s}}}, \quad K_{t s} \simeq e^{i \sigma} \frac{m_{c}}{m_{t}}-e^{i \tau} \sqrt{\frac{m_{d}}{m_{b}}}, \quad K_{t b} \simeq e^{i \tau}+\frac{m_{c}}{m_{t}} \sqrt{\frac{m_{d}}{m_{b}}} e^{i \sigma} .
\end{aligned}
$$

In this case, $\sigma=81.09^{\circ}, \tau=-98.91^{\circ}$ are obtained to get a good experimental agreement of the $\left|K_{u s}\right|$ and $\left|K_{c d}\right|$ elements with their experimental values and then $\psi_{U}=\psi_{D}+81.09^{\circ}, \theta_{U}=\theta_{D}-180^{\circ}$. The obtained numerical values for the CKM matrix elements are in Table \. The CP violating phase is $\delta=82.18^{\circ}$ presenting a discrepancy of $26.79 \%$. From Table III notice that the error percentages of the magnitudes of the CKM matrix elements are lower than $10.00 \%$ with the exception of the corresponding to the $\left|K_{u b}\right|,\left|K_{c b}\right|$ and $\left|K_{t s}\right|$ elements which are equal to $118.31 \%, 18.50 \%$ and $16.81 \%$, respectively.

The corresponding $\xi^{U}$ and $\xi^{D}$ Yukawa coupling matrices in the mass basis for this case are given by,

$$
\begin{gathered}
\xi^{U} \simeq \frac{\sqrt{2}}{v}\left(\begin{array}{ccc}
\left(c^{(U)}-2 d^{(U)}\right) m_{u} & \left(d^{(U)}-c^{(U)}\right) \sqrt{m_{u} m_{c}} e^{i \psi_{U}} & a^{(U)} \frac{m_{u}}{m_{c}} \sqrt{m_{c} m_{t}} e^{i\left(\psi_{U}+\theta_{U}\right)} \\
\left(d^{(U)}-c^{(U)}\right) \sqrt{m_{u} m_{c}} e^{-i \psi_{U}} & c^{(U)} m_{c} & \left(b^{(U)}-a^{(U)}\right) \sqrt{m_{u} m_{t}} e^{i \theta_{U}} \\
a^{(U)} \frac{m_{u}}{m_{c}} \sqrt{m_{c} m_{t}} e^{-i\left(\psi_{U}+\theta_{U}\right)} & \left(b^{(U)}-a^{(U)}\right) \sqrt{m_{u} m_{t}} e^{-i \theta_{U}} & a^{(U)} m_{t}
\end{array}\right), \\
\xi^{D} \simeq \frac{\sqrt{2}}{v}\left(\begin{array}{ccc}
\left(c^{(D)}-2 d^{(D)}\right) m_{d} & \left(d^{(D)}-c^{(D)}\right) \sqrt{m_{d} m_{s}} e^{i \psi_{D}} & a^{(D)} \frac{m_{d}}{m_{s}} \sqrt{m_{s} m_{b}} e^{i\left(\psi_{D}+\theta_{D}\right)} \\
\left(d^{(D)}-c^{(D)}\right) \sqrt{m_{d} m_{s}} e^{-i \psi_{D}} & c^{(D)} m_{s} & \left(b^{(D)}-a^{(D)}\right) \sqrt{m_{d} m_{b}} e^{i \theta_{D}} \\
a^{(U)} \frac{m_{d}}{m_{s}} \sqrt{m_{s} m_{b}} e^{-i\left(\psi_{D}+\theta_{D}\right)} & \left(b^{(D)}-a^{(D)}\right) \sqrt{m_{d} m_{b}} e^{-i \theta_{D}} & a^{(D)} m_{b}
\end{array}\right) .
\end{gathered}
$$

From the expressions (36), we observe that the Yukawa couplings to the first and the second generation fermions have the same form that the predicted by the Fritzsch and the Du-Xing ansatze. The couplings to the third generation fermions are stronger than the predicted by the Du-Xing ansatz and weaker than the obtained by the Cheng and Sher ansatz. This fact has consequences in the phenomenology as we will show in section IV.

\section{E. Fukuyama-Nishiura ansatz (FNA)}

Recently the following mass matrix ansatz has been proposed for quarks and leptons [9]:

$$
\widetilde{M}=\left(\begin{array}{lll}
0 & A & A \\
A & B & C \\
A & C & B
\end{array}\right)
$$

This form has been originally used for leptons (neutrinos) in order to reproduce a nearly bi-maximal lepton mixing. Moreover the mass matrix $\widetilde{M}$ is diagonalized by a rotation matrix according to:

$$
O^{T} \widetilde{M} O=\operatorname{diag}\left(-m_{1}, m_{2}, m_{3}\right)
$$


where $m_{1}, m_{2}$ and $m_{3}$ correspond to the fermion masses with $m_{1} \ll m_{2} \ll m_{3}$. By taking into account that $t r(\widetilde{M})$ and $\operatorname{det}(\widetilde{M})$ are invariants and using the eigenvalues equation for $\widetilde{M}$, we get:

$$
A= \pm \sqrt{\frac{m_{1} m_{2}}{2}}, \quad B=\frac{1}{2}\left(m_{2}+m_{3}-m_{1}\right), \quad C=-\frac{1}{2}\left(m_{3}-m_{2}+m_{1}\right) .
$$

The case where $B-C$ takes its maximum value corresponds to the assignment type C. The assignment type $\mathrm{D}$ is obtained by exchanging $m_{2}$ and $m_{3}$ in the already mentioned type $\mathrm{C}(39)$. For the assignment type D,

$$
A= \pm \sqrt{\frac{m_{3} m_{1}}{2}}, \quad B=\frac{1}{2}\left(m_{3}+m_{2}-m_{1}\right), \quad C=\frac{1}{2}\left(m_{3}-m_{2}-m_{1}\right) .
$$

The assignments type $\mathrm{C}$ and $\mathrm{D}$ are used for down-type and up-type quarks, respectively. The rotation matrices which diagonalize $\widetilde{M}$ for the assignments type $\mathrm{C}$ and $\mathrm{D}$ are,

$$
O=\left(\begin{array}{ccc}
c & s & 0 \\
-\frac{s}{\sqrt{2}} & \frac{c}{\sqrt{2}} & -\frac{1}{\sqrt{2}} \\
-\frac{s}{\sqrt{2}} & \frac{c}{\sqrt{2}} & \frac{1}{\sqrt{2}}
\end{array}\right), \quad O^{\prime}=\left(\begin{array}{ccc}
c^{\prime} & 0 & s^{\prime} \\
-\frac{s^{\prime}}{\sqrt{2}} & -\frac{1}{\sqrt{2}} & \frac{c^{\prime}}{\sqrt{2}} \\
-\frac{s^{\prime}}{\sqrt{2}} & \frac{1}{\sqrt{2}} & \frac{c^{\prime}}{\sqrt{2}}
\end{array}\right)
$$

respectively, where

$$
\begin{array}{cc}
c=\cos \varphi=\sqrt{\frac{m_{2}}{m_{2}+m_{1}}}, & s=\sin \varphi=\sqrt{\frac{m_{1}}{m_{1}+m_{2}}}, \\
c^{\prime}=\cos \varphi^{\prime}= \pm \sqrt{\frac{m_{3}}{m_{1}+m_{3}}}, & s^{\prime}=\sin \varphi^{\prime}= \pm \sqrt{\frac{m_{1}}{m_{1}+m_{3}}} .
\end{array}
$$

In order to get a good agreement of $\frac{1}{9} \sum_{i=1}^{9}\left|K_{i j}\right|$ with their experimental values, we get $\sigma=-180^{\circ}$ and $\tau=4.84^{\circ}$. From this result, the numerical values corresponding to the updated magnitudes of the CKM matrix elements for the Fukuyama-Nishiura ansatz are obtained [9] (see Table \).

From Table III, there are a very good agreement of the CKM matrix elements with their experimental values since the corresponding error percentages are lower than $2 \%$. The exceptions are the $\left|K_{u b}\right|$ and $\left|K_{t d}\right|$ elements, which present a discrepancies of about $28.72 \%$ and $18.80 \%$ respect to its corresponding experimental magnitudes. It is also important to point out that the Fukuyama-Nishiura ansatz leads to a much better prediction of the CKM matrix elements than the obtained from the Fritzsch and the Du-Xing ansatze. The $C P$ violating phase $\delta=-87.55^{\circ}$ in this case is inconsistent to its experimental value because it presents a discrepancy of about $168.72 \%$. Furthermore $\theta_{U}=\theta_{D}+184.84$ and $\psi_{U}=\psi_{D}-4.84^{\circ}$ are obtained.

Finally, the Yukawa coupling matrices in the mass basis are given by

$$
\begin{aligned}
& \xi^{U} \simeq \frac{\sqrt{2}}{2 v}\left(\begin{array}{ccc}
\left(b^{(U)}+c^{(U)}-4 a^{(U)}\right) m_{u} & 0 & f^{(U)} \sqrt{m_{u} m_{t}} e^{i\left(\psi_{U}+\theta_{U}\right)} \\
0 & \left(b^{(U)}-c^{(U)}\right) m_{t}+\left(b^{(U)}+c^{(U)}\right) m_{c} & 0 \\
f^{(U)} \sqrt{m_{u} m_{t}} e^{-i\left(\psi_{U}+\theta_{U}\right)} & 0 & \left(b^{(U)}+c^{(U)}\right) m_{t}
\end{array}\right) \\
& \xi^{D} \simeq \frac{\sqrt{2}}{2 v}\left(\begin{array}{ccc}
2\left(b^{(D)}-2 a^{(D)}\right) m_{d} & \left(b^{(D)}-c^{(D)}\right) \sqrt{\frac{m_{d}}{m_{s}}} m_{b} e^{i \psi_{D}} & 0 \\
\left(b^{(D)}-c^{(D)}\right) \sqrt{\frac{m_{d}}{m_{s}}} m_{b} e^{-i \psi_{D}} & \left(b^{(D)}-c^{(D)}\right) m_{b}+\left(b^{(D)}+c^{(D)}\right) m_{s} & 0 \\
0 & 0 & \left(b^{(D)}+c^{(D)}\right) m_{b}
\end{array}\right) .
\end{aligned}
$$

where $f^{(U)}=\left(2 a^{(U)}+b^{(U)}-c^{(U)}\right)$. From the above expressions, the couplings between the neutral Higgs field $h^{0}$ and the quarks pairs $u-c, c-t, d-b$ and $s-b$ are vanishing, which it is meaning that there are no flavor changing neutral currents involving terms like $h^{0} \bar{u} c, h^{0} \bar{c} t, h^{0} \bar{d} b, h^{0} \bar{s} b$.

\section{F. Non-mixing Top quark Ansatz (NTA)}

We propose the following mass matrix ansatz for up-type quarks

$$
\widetilde{M}=\left(\begin{array}{ccc}
B & C & 0 \\
C & D & 0 \\
0 & 0 & A
\end{array}\right)
$$


which is diagonalized by a rotation matrix $O$ given by

$$
O=\left(\begin{array}{ccc}
c_{\phi} & -s_{\phi} & 0 \\
s_{\phi} & c_{\phi} & 0 \\
0 & 0 & 1
\end{array}\right)
$$

according to $O^{T} \widetilde{M} O=\operatorname{diag}\left(m_{1},-m_{2}, m_{3}\right)$. In this case the condition $\tan \phi=\sqrt{\frac{m_{2}}{m_{3}}}$ has been imposed and using the expressions

$$
\operatorname{det} \widetilde{M}=A\left(B D-C^{2}\right), \quad \operatorname{tr} \widetilde{M}=B+D=-m_{2}+m_{1}
$$

it is obtained that

$$
A=m_{3}, \quad B=\frac{m_{1} m_{3}-m_{2}^{2}}{m_{2}+m_{3}}, \quad C=\frac{m_{1}+m_{2}}{m_{2}+m_{3}} \sqrt{m_{2} m_{3}}, \quad D=m_{2} \frac{m_{1}-m_{3}}{m_{2}+m_{3}},
$$

and therefore, the rotation matrix is

$$
O=\left(\begin{array}{ccc}
\sqrt{\frac{m_{3}}{m_{2}+m_{3}}} & -\sqrt{\frac{m_{2}}{m_{2}+m_{3}}} & 0 \\
\sqrt{\frac{m_{2}}{m_{2}+m_{3}}} & \sqrt{\frac{m_{3}}{m_{2}+m_{3}}} & 0 \\
0 & 0 & 1
\end{array}\right)
$$

By using the mass matrix ansatz given by (43) for the up-type quarks and the assignment type B of the Du-Xing ansatz for down-type quarks (22), the following CKM matrix elements are obtained

$$
\begin{array}{ccc}
K_{u d} \simeq c_{U}-s_{U} \sqrt{\frac{m_{d}}{m_{s}}} e^{i \sigma}, \quad K_{u s} \simeq c_{U} \sqrt{\frac{m_{d}}{m_{s}}}+s_{U} e^{i \sigma}, & K_{u b} \simeq c_{U} \sqrt{\frac{m_{d}^{2} m_{s}}{m_{b}^{3}}+s_{U} \sqrt{\frac{m_{d}}{m_{b}}} e^{i \sigma}} \\
K_{c d} \simeq-s_{U}-c_{U} \sqrt{\frac{m_{d}}{m_{s}}} e^{i \sigma}, \quad K_{c s} \simeq c_{U} e^{i \sigma}-s_{U} \sqrt{\frac{m_{d}}{m_{s}}}, & K_{c b} \simeq c_{U} \sqrt{\frac{m_{d}}{m_{b}}} e^{i \sigma}-s_{U} \sqrt{\frac{m_{d}^{2} m_{s}}{m_{b}^{3}}} \\
K_{t d} \simeq \sqrt{\frac{m_{d}^{2}}{m_{s} m_{b}}} e^{i \tau}, \quad K_{t s} \simeq-e^{i \tau} \sqrt{\frac{m_{d}}{m_{b}}}, & K_{t b} \simeq e^{i \tau},
\end{array}
$$

where:

$$
c_{U}=\sqrt{\frac{m_{t}}{m_{c}+m_{t}}}, \quad s_{U}=\sqrt{\frac{m_{c}}{m_{c}+m_{t}}}
$$

In order to get the best agreement of the $\left|K_{u s}\right|$ and $\left|K_{c d}\right|$ elements with their experimental values, we obtain $\sigma=-99.24^{\circ}$. On the other hand, by adjusting the $\tau$ parameter in order to get the best agreement of $\Im m\left(K_{t s}\right)$ with its experimental value, $\tau=90.00^{\circ}$ is gotten. Then, we obtain the magnitudes of the CKM matrix elements shown in Table [. Moreover, for this case we have $\psi_{U}=\psi_{D}-99.24^{\circ}, \theta_{U}=\theta_{D}+189.24^{\circ}$. And for the $C P$ violating phase we obtain $\delta=80.68^{\circ}$. This result roughly agrees with the experimental value, presenting a discrepancy of about $25.43 \%$.

The Table I shows the error percentages of the magnitudes of the Cabbibo-Kobayashi-Maskawa matrix elements and $\mathrm{CP}$ violating phase $\delta$ respect to their experimental values. In this Table we observe that the error percentages of the magnitudes of the CKM matrix elements are lower than $11.00 \%$ with the exception of the corresponding to the $\left|K_{u b}\right|,\left|K_{c b}\right|$ and $\left|K_{t s}\right|$ elements which are equal to $108.64 \%, 32.01 \%$ and $29.91 \%$, respectively. For this reason, the Cabbibo-Kobayashi-Maskawa matrix obtained by using the above prescription exhibits a good agreement with the experimental results. 
According to the mass matrix structure given by (43), we propose the following ansatz for the Yukawa coupling matrix in the flavor basis:

$$
\xi^{U, 0}=\frac{\sqrt{2}}{v}\left(\begin{array}{ccc}
b^{(U)} B_{U} & c^{(U)} C_{U} e^{i \psi_{U}} & 0 \\
c^{(U)} C_{U} e^{-i \psi_{U}} & d^{(U)} D_{U} & 0 \\
0 & 0 & a^{(U)} A_{U}
\end{array}\right)
$$

Hence, when the new ansatz and type B assignment of the Du-Xing ansatz are used for the quarks $U$ and $D$ respectively, the Yukawa couplings matrices in the mass basis are given by:

$$
\begin{gathered}
\xi^{U}=\frac{\sqrt{2}}{v}\left(\begin{array}{ccc}
b^{(U)} m_{u} & \left(c^{(U)}-d^{(U)}\right) \frac{m_{c}}{m_{t}} \sqrt{m_{c} m_{t}} e^{i \psi_{U}} & 0 \\
\left(c^{(U)}-d^{(U)}\right) \frac{m_{c}}{m_{t}} \sqrt{m_{c} m_{t}} e^{-i \psi_{U}} & -d^{(U)} m_{c} & 0 \\
0 & 0 & a^{(U)} m_{t}
\end{array}\right), \\
\xi^{D} \simeq \frac{\sqrt{2}}{v}\left(\begin{array}{ccc}
\left(c^{(D)}-2 d^{(D)}\right) m_{d} & \left(d^{(D)}-c^{(D)}\right) \sqrt{m_{d} m_{s}} e^{i \psi_{D}} & a^{(D) \frac{m_{d}}{m_{s}} \sqrt{m_{s} m_{b}} e^{i\left(\psi_{D}+\theta_{D}\right)}} \\
\left(d^{(D)}-c^{(D)}\right) \sqrt{m_{d} m_{s}} e^{-i \psi_{D}} & c^{(D)} m_{s} & \left(b^{(D)}-a^{(D)}\right) \sqrt{m_{d} m_{b}} e^{i \theta_{D}} \\
a^{(U)} \frac{m_{d}}{m_{s}} \sqrt{m_{s} m_{b}} e^{-i\left(\psi_{D}+\theta_{D}\right)} & \left(b^{(D)}-a^{(D)}\right) \sqrt{m_{d} m_{b}} e^{-i \theta_{D}} & a^{(D)} m_{b}
\end{array}\right) .
\end{gathered}
$$

From the previous expressions, the couplings between the neutral Higgs field $h^{0}$ and the quarks pairs $u$ - $t, c-t$ are vanishing, which implies that there are no flavor changing neutral currents involving terms of the form $h^{0} \bar{u} t, h^{0} \bar{c} t$.

\section{TWO BODY DECAYS OF THE TOP QUARK, $h^{0}$ AND $H^{ \pm}$}

Consider the top quark decay to an up-type quark plus the lightest neutral Higgs boson $h^{0}$. The interest on FCNC is expected to increase since this issue will be examined at both the LHC and ILC, where they hope to reach sensibilities of the order of $B\left(t \rightarrow q h^{0}\right) \gtrsim 10^{-5}$. In the framework of the SM, the branching fractions are strongly suppressed $B\left(t \rightarrow c h^{0}\right) \sim 10^{-15}$ and $B\left(t \rightarrow u h^{0}\right) \sim 10^{-17}$. In the framework of the 2HDM type III an enhancement is expected due to the FCNC presence at tree level. The decay width takes the form

$$
\Gamma\left(t \rightarrow q h^{0}\right)=\frac{G_{F} m_{t}}{4 \sqrt{2} \pi}\left(1-\frac{m_{h}^{2}}{m_{t}^{2}}\right)^{2}\left|\lambda_{t q}^{U}\right|^{2} \cos ^{2} \alpha
$$

where $\lambda_{t q}^{U}$ is the coupling $t q h^{0}$. Therefore, there are two possible decays: $t \rightarrow u h^{0}$ and $t \rightarrow c h^{0}$. In figure 1 , the branching fraction $B\left(t \rightarrow c h^{0}\right)$ as a function of the lightest neutral Higgs boson mass for the Fritzsch ansatz is plotted and orders of $10^{-3}$ are gotten. In this Figure 1, a decrease of the branching ratio with the increase of the Higgs mass is observed, but notice that the branching ratio raise up increasing the magnitudes of the coefficients $a^{(U)}-b^{(U)}$ of the coupling $t c h^{0}$. And the branching ratio decrease with the reduction of the mixing angle $\alpha$ between the neutral Higgs fields $h^{0}$ and $H^{0}$. These coefficients and the mixing angle $\alpha$ were taken to be equal to $0.75,1.00$ and $\frac{\pi}{4}, \frac{\pi}{15}, \frac{4 \pi}{9}$, respectively. The branching fraction $B\left(t \rightarrow u h^{0}\right)$ is two orders of magnitude lower than $B\left(t \rightarrow c h^{0}\right)$ and its behavior is quite similar.

In order to consider the different ansatze, the following ratio is useful,

$$
R^{(A / F)}=\frac{B\left(t \rightarrow q h^{0}\right)_{A}}{B\left(t \rightarrow q h^{0}\right)_{F}}=\left|\frac{\lambda_{t q, A}^{U}}{\lambda_{t q, F}^{U}}\right|^{2}
$$

it is respect to the Fritzsch ansatz $(\mathrm{F})$, with $q=u, c$ and $A=D X A, F D X A, D X F A, \tilde{X} A, F N A, N T A$. It does not depend on $m_{t}, m_{h}$ or $\cos \alpha$, it depends on the explicit form of the masses in the different kind of ansatze discussed. This ratio is typically of the order of $10^{-3}$ but in the case of the FNA and NTA such a kind of top quark decay width is zero because the top quark is completely decoupled of the two lighter generations.

Now, turn on the attention to the lightest neutral Higgs boson $h^{0}$ decays. The $h^{0}$ decays to the quark pairs $d \bar{b}$, $s \bar{b}, d \bar{s}$ and $b \bar{b}$ are considered. In the framework of the SM, the channel $h_{S M}^{0} \rightarrow b \bar{b}$ is the most studied channel because it plays a central role for its possible detection in the range $120-140 \mathrm{GeV}$ and its signature will be clear 


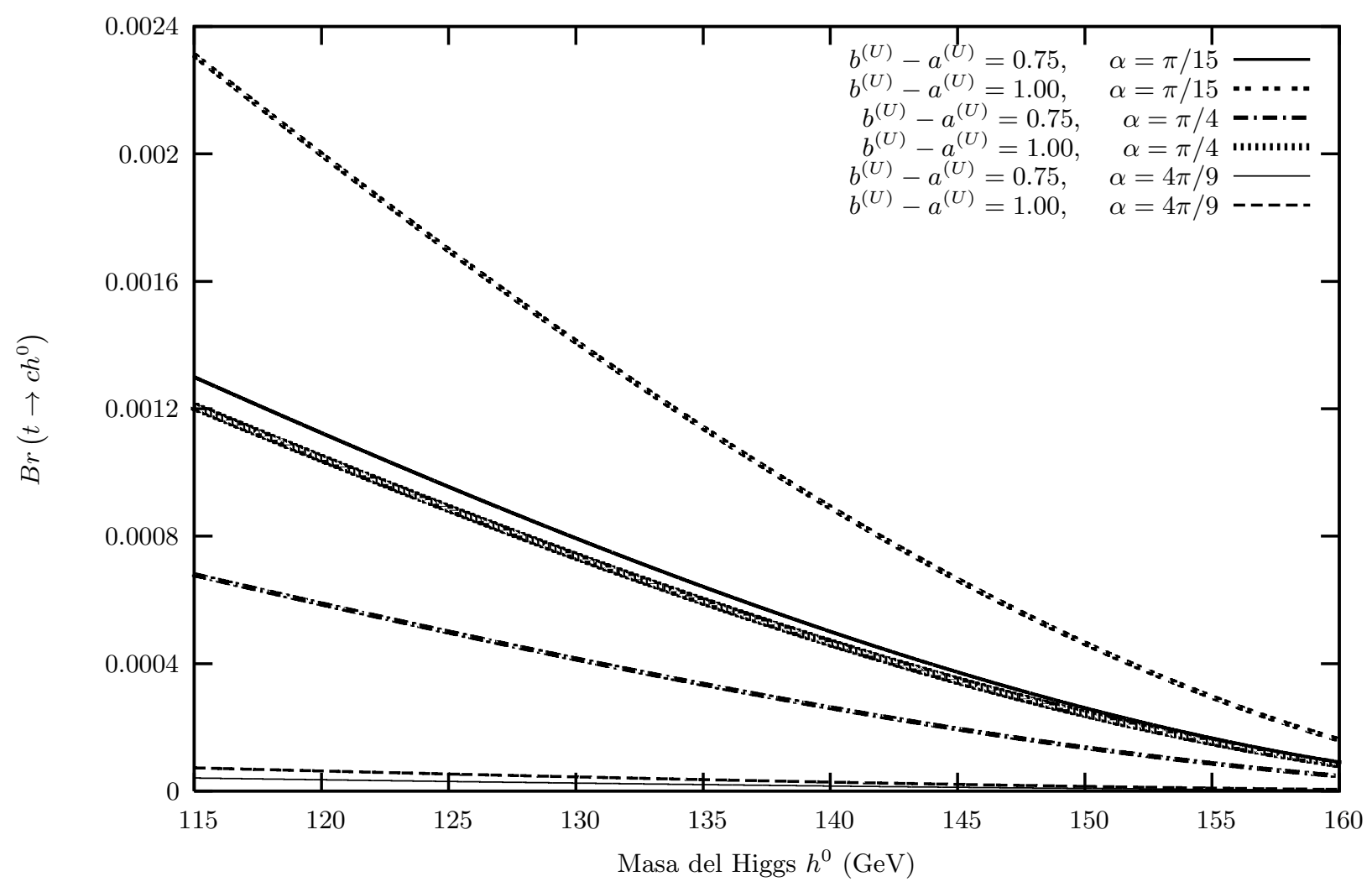

FIG. 1: Branching ratio $B\left(t \rightarrow c h^{0}\right)$ for the process $t \rightarrow c h^{0}$ in the Fritzsch ansatz and using different values of the Yukawa coupling parameters and $\alpha$.

enough. On the other hand, decays like $h^{0} \rightarrow s \bar{b}$ are interesting to study because FCNC in the Higgs sector would be a clear evidence of physics beyond the SM. In order to look for differences, the ratio $r_{b b}=\frac{\Gamma\left(h^{0} \rightarrow b \bar{b}\right)_{A}}{\Gamma\left(h^{0} \rightarrow b \bar{b}\right)_{S M}}$ between the decay widths $\Gamma\left(h^{0} \rightarrow b \bar{b}\right)_{A}$ and $\Gamma\left(h^{0} \rightarrow b \bar{b}\right)_{S M}$ is defined. Since for all ansatze considered in this work, the Yukawa couplings of the neutral Higgs boson $h^{0}$ and the quark pair $b \bar{b}$ have the same intensity, the ratio $r_{b b}$ has the same value for all ansatze. However, the ratio $r_{b b}$ gets the maximum value when $\cot \alpha=-a^{D}$, then for $\alpha=-\pi / 4$ and $a^{D}=1$, the ratio is 2 , its mean that the decay width for these ansatze can be twice the decay width predicted by the SM.

Another possible definition is the ratio, $\gamma_{s b}^{(A)}=\frac{\Gamma\left(h^{0} \rightarrow s \bar{b}\right)_{A}}{\Gamma\left(h^{0} \rightarrow b \bar{b}\right)_{A}}$. From Table III the relative decay widths for the process $h^{0} \rightarrow s \bar{b}$ predicted by the Fritzsch ansatz are at least one order of magnitude bigger than the corresponding to the assignments type A and B of the Du-Xing ansatz. This is due to the fact that the intensity of the Yukawa couplings to the third generation fermions for the Fritzsch ansatz is stronger than the one corresponding to the assignments type A and B of the Du-Xing ansatz, which implies that the Fritzsch ansatz leads to a bigger probability of finding the decay processes $h^{0} \rightarrow s \bar{b}$ than the obtained by these assignments of the Du-Xing ansatz.

Now, about the charged Higgs boson decays, two cases are considered: a charged Higgs boson lighter than the top quark, $m_{H}=150 \mathrm{GeV}$ and a heavier charged Higgs boson $m_{H}=250 \mathrm{GeV}$. In the first case, the top quark could decay into a charged Higgs boson and it will be an alternative to the usual channel $t \rightarrow W^{+} b$. Taking a light charged Higgs boson, the interesting channels to detect a Higgs boson would be $H^{+} \rightarrow c \bar{s}, H^{+} \rightarrow c \bar{b}$ and $t \rightarrow H^{+} b$. The decay widths were evaluated using phases different of zero, $\psi_{D}=\theta_{D}=\frac{\pi}{18}$ but we also did it with phases equal to zero and the results do not change in order of magnitude. In table IV the results are shown, notice that the channel $H^{+} \rightarrow c \bar{b}$ could compete with the searched channel $H^{+} \rightarrow c \bar{s}$, however in the FNA and NTA ansatze the $c \bar{s}$ channel is bigger than the $c \bar{b}$ channel. About the option $t \rightarrow H^{+} b$ the branching fraction is of the order of $10^{-1}$ in the different ansatze considered. 


\begin{tabular}{|c|c|c|c|}
\hline & $\alpha=\frac{\pi}{3}$ & $\alpha=\frac{\pi}{18}$ & $\alpha=\frac{4 \pi}{9}$ \\
\hline \hline$\gamma_{s b}^{(F A)}$ & $7.29 \times 10^{-2}$ & $2.88 \times 10^{-2}$ & $8.95 \times 10^{-4}$ \\
\hline$\gamma_{s b}^{(D X A)}$ & $7.10 \times 10^{-4}$ & $5.61 \times 10^{-4}$ & $4.71 \times 10^{-5}$ \\
\hline$\gamma_{s b}^{(F D X)}$ & $7.10 \times 10^{-4}$ & $5.61 \times 10^{-4}$ & $4.71 \times 10^{-5}$ \\
\hline$\gamma_{s b}^{(D X F A)}$ & $7.29 \times 10^{-2}$ & $2.88 \times 10^{-2}$ & $8.95 \times 10^{-4}$ \\
\hline$\gamma_{s b}^{(\tilde{X} A)}$ & $1.92 \times 10^{-3}$ & $1.51 \times 10^{-3}$ & $4.71 \times 10^{-5}$ \\
\hline \hline
\end{tabular}

TABLE III: Relative decay width $\gamma_{s b}^{(A)}=\frac{\Gamma\left(h^{0} \rightarrow s \bar{b}\right)_{A}}{\Gamma\left(h^{0} \rightarrow b \bar{b}\right)_{A}}$ for the process $h^{0} \rightarrow s \bar{b}$ in the different ansatze with $M_{h^{0}}=120 G e V$ and the Yukawa coupling coefficients of the order of one.

In the case of a charged Higgs boson heavier than the top quark, $\Gamma\left(H^{+} \rightarrow t \bar{b}\right), \Gamma\left(H^{+} \rightarrow t \bar{s}\right), \Gamma\left(H^{+} \rightarrow t \bar{d}\right)$, $\Gamma\left(H^{+} \rightarrow c \bar{s}\right)$ and $\Gamma\left(H^{+} \rightarrow c \bar{b}\right)$ are evaluated and they are shown in Table $\mathrm{V}$. It is worth to notice that the most important channel would be $H^{+} \rightarrow t \bar{b}$ except for the FNA where it is $H^{+} \rightarrow c \bar{s}$.

\begin{tabular}{|c|c|c|c|c|c|c|c|}
\hline$\Gamma(\mathrm{GeV})$ & FA & DXA & FDXA & DXFA & $\tilde{X}$ A & FNA & NTA \\
\hline$\Gamma\left(H^{+} \rightarrow c \bar{s}\right)$ & $4.83 \times 10^{-4}$ & $3.77 \times 10^{-5}$ & $6.11 \times 10^{-5}$ & $3.34 \times 10^{-5}$ & $3.49 \times 10^{-5}$ & 2.81 & $3.72 \times 10^{-5}$ \\
\hline$\Gamma\left(H^{+} \rightarrow c b\right)$ & $1.04 \times 10^{-2}$ & $3.87 \times 10^{-5}$ & $1.06 \times 10^{-2}$ & $1.12 \times 10^{-4}$ & $4.41 \times 10^{-5}$ & $5.28 \times 10^{-3}$ & $3.15 \times 10^{-6}$ \\
\hline$B\left(t \rightarrow H^{+} b\right)$ & $1.22 \times 10^{-1}$ & $1.24 \times 10^{-1}$ & $1.24 \times 10^{-1}$ & $1.24 \times 10^{-1}$ & $1.24 \times 10^{-1}$ & $1.24 \times 10^{-1}$ & $1.24 \times 10^{-1}$ \\
\hline
\end{tabular}

TABLE IV: The decay widths $\Gamma\left(H^{+} \rightarrow c \bar{s}\right), \Gamma\left(H^{+} \rightarrow c \bar{b}\right)$ and the fraction $B\left(t \rightarrow H^{+} b\right)$ using the mass matrices ansatze discussed and setting up $M_{H^{+}}=150 \mathrm{GeV}, \psi_{D}=\theta_{D}=\frac{\pi}{18}$

\begin{tabular}{|c|c|c|c|c|c|c|c|}
\hline$\Gamma(\mathrm{GeV})$ & FA & DXA & FDXA & DXFA & XA & FNA & NTA \\
\hline$\Gamma\left(H^{+} \rightarrow c \bar{s}\right)$ & $8.06 \times 10^{-4}$ & $6.29 \times 10^{-5}$ & $1.02 \times 10^{-4}$ & $5.57 \times 10^{-5}$ & $5.82 \times 10^{-5}$ & 4.68 & $6.21 \times 10^{-5}$ \\
\hline$\Gamma\left(H^{+} \rightarrow c b\right)$ & $1.74 \times 10^{-2}$ & $6.45 \times 10^{-5}$ & $1.76 \times 10^{-2}$ & $1.87 \times 10^{-4}$ & $7.35 \times 10^{-5}$ & $8.80 \times 10^{-3}$ & $5.26 \times 10^{-6}$ \\
\hline$\Gamma\left(H^{+} \rightarrow t b\right)$ & 1.32 & 1.34 & 1.34 & 1.34 & 1.34 & 1.34 & 1.34 \\
\hline$\Gamma\left(H^{+} \rightarrow t \bar{s}\right)$ & $6.12 \times 10^{-2}$ & $4.57 \times 10^{-4}$ & $7.71 \times 10^{-3}$ & $2.64 \times 10^{-2}$ & $1.38 \times 10^{-3}$ & $2.23 \times 10^{-3}$ & $1.38 \times 10^{-3}$ \\
\hline$\Gamma\left(H^{+} \rightarrow t \bar{d}\right)$ & $2.69 \times 10^{-3}$ & $2.71 \times 10^{-5}$ & $6.44 \times 10^{-4}$ & $1.41 \times 10^{-3}$ & $6.90 \times 10^{-5}$ & $2.03 \times 10^{-4}$ & $7.24 \times 10^{-5}$ \\
\hline \hline
\end{tabular}

TABLE V: The decay widths $\Gamma\left(H^{+} \rightarrow c \bar{s}\right), \Gamma\left(H^{+} \rightarrow c \bar{b}\right), \Gamma\left(H^{+} \rightarrow t \bar{b}\right), \Gamma\left(H^{+} \rightarrow t \bar{s}\right)$ and $\Gamma\left(H^{+} \rightarrow t \bar{d}\right)$ in the different ansatze using $M_{H^{+}}=250 \mathrm{GeV}$ and $\psi_{D}=\theta_{D}=\frac{\pi}{18}$.

\section{CONCLUSIONS}

The quark mass matrices ansatze proposed by Fritzsch, Du-Xing and Fukuyama-Nishiura [1, 9, 14] have been reviewed in the framework of the general two Higgs doublet model and the corresponding Yukawa matrices in the flavor basis. For these ansatze, the numerical values of the CKM matrix elements and their experimental values have been compared, obtaining that the Fukuyama-Nishiura ansatz leads to the best agreement with the experimental results. The CKM matrix elements by combining the Fritzsch and the Du-Xing ansatze for the up-type and downtype quarks in different ways have also been obtained. For the CKM matrix obtained by using the Fritzsch and the Du-Xing ansatze for the $U$-type and $D$-type quarks respectively a better agreement with the experimental CKM matrix elements is gotten than the resulting when other Fritzsch and Du-Xing ansatze combinations are used. Moreover, the CKM matrix by using two different assignments of the Du-Xing ansatz for the $U$-type and $D$-type quarks has been computed, obtaining a very good consistency between the magnitudes of six of their elements and their experimental values. In the concerning to the Fukuyama-Nishiura ansatz, the Yukawa coupling matrices in the mass basis for both types of quarks have been computed by using two different assignments for the $U$-type and $D$-type quarks. In this case, vanishing entrances of the $\xi^{U}$ and $\xi^{D}$ matrices are obtained, they are implying the absence of the flavor changing neutral currents involving terms of the form $h^{0} \bar{u} c, h^{0} \bar{c} t, h^{0} \bar{d} b, h^{0} \bar{s} b$. Finally, a new type of ansatz has been proposed where the FCNCs involving the top quark have vanished completely and an excelent agreement with the CKM experimental elements is gotten, with the exception of the $\left|K_{u b}\right|,\left|K_{c b}\right|$ and $\left|K_{t d}\right|$ elements. Results are shown in Tables \and II]

On the other hand, a discussion about the phenomenology of the two body decays of the lightest Higgs boson, the top quark and the charged Higgs boson using the different mass matrices ansatze in the framework of the 2HDM type 
III is presented in section [V] and results are shown in tables III, IV and V] For the lightest neutral Higgs boson, the decays $h^{0} \rightarrow b \bar{b}$ and $h^{0} \rightarrow b \bar{s}$ are interesting. In the different ansatze considered the channel $b \bar{b}$ can be enhanced respect to the SM using appropriate values of the Yukawa parameters. And the channel $b \bar{s}$ would be more important in the Fritzsch ansatz than in the other ansatze. About the charged Higgs decays two options have been explored: a lighter charged Higgs boson an a heavier one than top quark. Taking a lighter charged Higgs boson is possible to get a bigger decay width for $\mathrm{H}^{+} \rightarrow c \bar{b}$ than the decay width for $\mathrm{H}^{+} \rightarrow c \bar{s}$ depending on the used ansatz. And in the case of a heavier charged Higgs boson than the top quark, if the Fukuyama-Nishiura ansatz is used, the most important channel would be $H^{+} \rightarrow c \bar{s}$. Also in the case of a heavier charged Higgs boson than the top quark, the channels with a top quark in the final state would be relevant in a eventual search of the charged Higgs boson.

We acknowledge to R. Diaz for useful discussions. This work has been supported by COLCIENCIAS and Fundación Banco de la Republica.

\section{APPENDIX A}

\section{CKM mixing matrix}

The corresponding standard parametrization of the CKM mixing matrix is [21],

$$
K=\left(\begin{array}{ccc}
c_{12} c_{13} & s_{12} c_{13} & s_{13} e^{-i \delta} \\
-s_{12} c_{23}-c_{12} s_{23} s_{13} e^{i \delta} & c_{12} c_{23}-s_{12} s_{23} s_{13} e^{i \delta} & s_{23} c_{13} \\
s_{12} s_{23}-c_{12} c_{23} s_{13} e^{i \delta} & -c_{12} s_{23}-s_{12} c_{23} s_{13} e^{i \delta} & c_{23} c_{13}
\end{array}\right)
$$

with $c_{i j}=\cos \theta_{i j}, s_{i j}=\sin \theta_{i j} \operatorname{con} i, j=1,2,3$, and $\delta$ the CP violating phase.

\section{Quark masses}

In the numerical computations, we used the central values of the following quark masses at the energy scale of the order of $m_{Z}[21]$ :

$$
\begin{array}{cr}
m_{u}\left(m_{Z}\right)=1.64 \pm 0.40 \mathrm{MeV}, \quad m_{c}\left(m_{Z}\right)=620 \pm 30 \mathrm{MeV}, \quad m_{t}\left(m_{Z}\right)=172.7 \pm 2.9 \mathrm{GeV} \\
m_{d}\left(m_{Z}\right)=2.92 \pm 0.60 \mathrm{MeV}, \quad m_{s}\left(m_{Z}\right)=55.56 \pm 8.00 \mathrm{MeV}, \quad m_{b}\left(m_{Z}\right)=2.85 \pm 0.18 \mathrm{GeV}
\end{array}
$$

\section{REFERENCES}

[1] H. Fritzsch, Phys. Lett. B 70, 436 (1977); Phys. Lett. B 73, 317 (1978); Nucl. Phys. B 155, 189 (1979); H. Fritzsch and J. Plankl, Phys. Lett. B 237, 451 (1990).

[2] H. Fritzsch and Z. Xing, Phys. Lett. B 555, 63 (2003) arXiv:hep-ph/0212195; Prog. Part. Nucl. Phys. 45, 1 (2000) arXiv:hep-ph/9912358; Nucl. Phys. B 556, 49 (1999) arXiv:hep-ph/9904286; Phys. Lett. B 353, 114 (1995) arXiv:hep-ph/9502297.

[3] L. Lavoura, Int JP A 9, 1873 (1994).

[4] Y. F. Zhou, J. Phys. G 30, 783 (2004) arXiv:hep-ph/0307240; arXiv:hep-ph/0309076.

[5] G. F. Giudice, Mod. Phys. Lett. A 7, 2429 (1992) arXiv:hep-ph/9204215].

[6] T. Fukuyama, H. Nishiura, Proceedings of 1997 Shizuoka Workshop of masses and Mixings of Quarks and leptons, 19-21 (1997) arXiv:hep-ph/9702253

[7] D. Cremades, L.E. Ibanez, F. Marchesano, JHEP 0307, 038 (2003); D. Cremades, L.E. Ibanez, F. Marchesano, JHEP 0405, 079 (2004).

[8] P. Ramond, R. G. Roberts and G. G. Ross, Nucl. Phys. B406, 19 (1993); G. C. Branco, L. Lavoura and F. Mota, Phys. Rev. D39, 3443 (1989).

[9] S. Dimopoulos, J. L. Hall and S. Raby, OSU, preprint-DOE-ER-01545-567 (91); H. Georgi and C. Jarlskog, Phys. Lett. B86, 297 (1979); K. Matsuda and H. Nishiura, Phys. Rev. D 69, 053005 (2004) arXiv:hep-ph/0309272. K. Matsuda, T. Fukuyama and H. Phys. Rev. D 61, 053001 (2000) arXiv:hep-ph/9906433.

[10] T. P. Cheng, M. Sher, Phys. Rev. D 35, 3484 (1987).

[11] G. C. Branco, D. Emmanuel-Costa, R. Gonzalez Felipe, Phys. Lett. B 477, 147-155, (2000); G. C. Branco, M. N. Rebelo and J. Silva-Marcos, Phys. Lett. B 597155 (2004) arXiv:hep-ph/0403016; G. C. Branco, L. Lavoura, J. Silva, CP Violation, Clarendon Press, Oxford (1999). 
[12] C. Jarlskog, Phys. Rev. Lett. 55, 1039 (1985).

[13] S. N. Gupta, J. M. Johnson, Phys. Rev. D 44, 2110 (1991).

[14] D. Du and Z.Z. Xing, Phys. Rev.D 48, 2349 (1993); H. Fritzsch and Z.Z. Xing, Phys. Lett. B 353, 114 (1995).

[15] Y. Koide, H. Nishiura, K. Matsuda, T. Kikuchi and T. Fukuyama, Phys. Rev. D 66, 093006 (2002) arXiv:hep-ph/0209333.

[16] N. Haba and K. Yoshioka, Nucl. Phys. B 739, 254 (2006) arXiv:hep-ph/0511108; T. Fukuyama, H. Nishiura, arXiv:hep-ph/9702253; A. Datta and P. J. O'Donnell, Phys. Rev. D 72, 113002 (2005) arXiv:hep-ph/0508314]; K. Kang and S. K. Kang, Phys. Rev. D 56, 1511 (1997) arXiv:hep-ph/9704253.

[17] H. Nishiura, K. Matsuda, T. Kikuchi and T. Fukuyama, Phys. Rev. D 65, 097301 (2002) arXiv:hep-ph/0202189.

[18] S. Glashow and S. Weimberg. Phys. Rev. D D15, 1958 (1977).

[19] Y. L. Wu, L. Wolfenstein, Phys. Rev. Lett. 73, 1762 (1994);W.S. Hou, Phys. Lett. B 296179 (1993); R. Diaz, R. Martinez and J. A. Rodriguez, Phys. Rev. D 63, 095007 (2001);Phys. Rev. D 64, 033004 (2001); Phys. Rev. D 67, 075011 (2003)

[20] J. F. Gunion and H. E. Haber, Phys. Rev. D 67, 075019 (2003) arXiv:hep-ph/0207010; Phys. Rev. D 72, 095002 (2005) arXiv:hep-ph/0506227.

[21] Particle Data Group: G.-M. Yao, et al (2006); J. Phys G 33, 1 (2006)

[22] L.-L. Chau and W.-Y. Keung, Phys. Rev. Lett 53, 1802 (1984).

[23] H. Harari and M. Leurer, Phys. Lett. B 181, 123 (1986).

[24] F. J. Botella and L.-L. Chao, Phys. Lett. B 168, 97 (1986).

[25] H. Fusaoka and Y. Koide, Phys. Rev. D 57, 3986 (1998) arXiv:hep-ph/9712201. 